\title{
DREAM Project: Decreasing REcurrent pain and Anxiety in Medical procedures on a pediatric population
}

Sylvie Le May 1, David Paquin 2, Stéphane Bouchard 3, Jean-Simon Fortin 4 and Christelle Khadra 1

1 Université de Montréal

2 Université du Québec en Abitibi-Témiscamingue

3 Université du Québec en Outaouais

4 CHU Sainte-Justine

Abstract - The DREAM project is a joint venture between the medical world, university professors and de velopers with one goal in mind: create an efficient distraction tool to reduce pain and anxiety in children receiving medical treatments. Growing evidence in the medical literature suggests that virtual reality is a very potent and effective way of creating distraction. In turn, distraction has been shown to reduce pain and anxie ty in children who are receiving painful medical procedures. The pediatric population offers quite a challenge when it comes to pain control, especially the pain caused by medical procedure. Anxiety and anticipation greatly modulates pain perception and can often jeopardize a treatment if a child's collaboration cannot be obtained. With those premises in mind, our team is trying to create an efficient virtual reality game for distracting kids from their pain. The DREAM game has been tailored for the very specific needs and characteristics of our target population. The experience is optimized for the oculus rift and the controls have been simplified to work in various medical settings with minimal preparation time. Our main concern was to create a game that delivers a potent distractive effect while inciting almost no side effects or cyber sickness. The DREAM project is currently being tested on children with burn injuries. The objective of this paper is to present the methodology used to validate our hypotheses.

Index Terms - Virtual Reality; Distraction; Video Games; Pain; Anxiety; Burn injuries; Pediatric population.

\section{INTRODUCTION}

Children with burn injuries experience severe pain during procedures despite the fact that doses of analgesics used with this population has almost doubled since 1992 (Ratcliff SL, Brown A, Rosenberg L, Rosenberg M, Robert RS, Cuervo LJ 2006). Burn injuries are the leading cause of morbidity and mortality in children (Sharma RK, Parashar A 2010). Procedural pain related to dressing changes is difficult to assess and manage in burn patients and can impede recovery, cause high levels of anxiety and alter future pain behaviors, 
resulting at times in chronic pain, paresthesia, posttraumatic stress disorder or even depression (von Baeyer CL, Tupper SM 2010 - Gandhi M, Thomson C, Lord D, Enoch S 2010). Pain can also decrease participation in activities such as physiotherapy resulting in poor health outcomes (von Baeyer CL, Tupper SM 2010).

Procedural pain management still heavily relies on opioids, benzodiazepines and other pharmacological agents which cause a lot of side effects and do not always provide children with sufficient pain reduction (Ratcliff SL, Brown A, Rosenberg L, Rosenberg M, Robert RS, Cuervo LJ 2006 Gandhi M, Thomson C, Lord D, Enoch S 2010 - Hoffman HG, Meyer WJ, 3rd, Ramirez M, Roberts L, Seibel EJ, Atzori B 2014). On the other hand, the effectiveness of multimodal approaches combining medication with non-pharmacological interventions for procedural pain relief has been highlighted in recent years (Uman LS, Birnie KA, Noel M, Parker JA, Chambers CT, McGrath PJ 2013). Distraction techniques are based on the gate control theory later expanded by McCaul and Mallot who suggest that the attention devoted to pain stimuli is limited and pain can therefore be modulated by diverting attention away from the noxious stimulus (Melzack R, Wall PD 1965 - Li A, Montaño Z, Chen VJ, Gold JI 2011). As a result, it is theorized that distraction techniques engaging multiple senses may grab the child's attention more than the techniques that only engage one sense.

Hence, the increasing interest in more immersive and interactive methods of distraction such as VR for the management of procedural pain in children. VR has gained increasing consideration as it engages multiple senses and allows interactions with a virtual world.

\section{AIM OF THE PROJECT}

Procedural pain management in children remains suboptimal (Harrison D, Joly C,
Chretien C, Cochrane S, Ellis J, Lamontagne C 2014) and the importance of developing more effective methods is required. Current guidelines on pediatric procedural pain management recommend the combination of non-pharmacological and pharmacological interventions to enhance pain management and decrease the numerous side effects of analgesics (Sharma RK, Parashar A 2010). Distraction, such as Virtual reality (VR), has been identified as one of the most effective non-pharmacological interventions. Despite the seeming utility of VR in pain management (Malloy KM, Milling LS 2010 - Hoffman HG, Chambers GT, Meyer WJ 2011), its use in healthcare settings was limited, mainly due to its high cost. However, there has been a breakthrough in 2014 when Facebook purchased the Oculus Rift (OR) for mass production allowing access to the latest technology in VR at a relatively low cost. Use of the $\mathrm{OR}$ has shown a promising effect on pain related to burn injuries in children but its effectiveness remains to be determined ( Hoffman HG, Meyer WJ, 3rd, Ramirez M, Roberts L, Seibel EJ, Atzori B 2014).

The overall goal of the DREAM project is to provide knowledge on immersive technologies and well adapted VR game design to control pain with the hope of providing a less traumatizing experience to children with burn injuries. The main objective of this study is to assess the effectiveness of VR distraction via the OR on procedural pain in children with burn injuries. The use of the OR is motivated by the fact that it's an affordable and powerful distraction tool that provides a wild field of view, high resolution display, integrated 3D audio, motion detection and low latency.

To our knowledge, no other pediatric hospital across Canada uses VR games as a method of pain management. This project will provide evidence-based knowledge on a non-pharmacological method of pain management through innovative interventions. In addition to providing this tool as a new alternative to pain 
management, our project innovates by using videogames where the gameplay (convergence zones, surprises, discoveries and activities, colors and interactive elements) or level of difficulty is tailored to children's developmental stages, as well as adapted to a hospital environment. This allows control over the components designed to maximize the feeling of immersion and distraction in the targeted audience and controlling for symptoms of cybersickness.

Since the technology is becoming an inherent part of children's lives, its integration into routine care requires rigorous studies to demonstrate its effectiveness. Indeed, VR game distraction via OR could be a highly effective method to relieve pain, anxiety and it is easy to use. We believe that it could reduce the need for opioids and anxiolytics in burn children and provide a less traumatizing hospital experience, while promoting a more humanistic care environment by combining immersive technologies to standard analgesic interventions administered to these children. The expected results will have a direct effect on physical (pain, comfort) and psychological (anxiety, pain memories) health of the child. In addition, clinical implications may include other indicators of quality of care and economic benefits such as duration of the dressing change, amount of opioids and anxiety drugs administered, use of coanalgesia, number of professionals required for the dressing change and duration of wound healing.

\section{FEASIBILITY}

VR is an active distraction method that allows the user to interact with an immersive environment generated by a computer stimulating different senses (vision, hearing and sometimes touch). VR allows a mental escape through multisensory interaction. In the past ten years, VR was tested through different studies for various painful procedures in children and adult populations and showed a tendency toward a beneficial effect on pain and anxiety (Hoffman HG,
Richards TL, Van Oostrom T, Coda BA, Jensen MP, Blough DK 2007 - Asl Aminabadi N, Erfanparast L, Sohrabi A, Ghertasi Oskouei S, Naghili A 2012). A study by Hoffman et al. in 2007, showed that the percentage of reduction in pain by VR was comparable to the reduction resulting from a moderate dose of hydromorphone, equivalent to what a patient would receive during a burn wound care (Hoffman HG, Chambers GT, Meyer WJ, 3rd, Arceneaux LL, Russell WJ, Seibel EJ 2011). Yet, the authors state that they believe the best analgesia is achieved by combining VR with pharmacological treatment. However, despite positive evaluations, the effectiveness of immersive technologies and well adapted VR game design hasn't been determined in children (Birnie KA, Noel M, Parker JA, Chambers CT, Uman LS, Kisely SR 2014 - Morris LD, Louw QA, Grimmer-Somers K 2009). Studies have either tested VR on experimental pain or on short needle-related procedures or within case studies limiting the statistical significance of the effect (Dahlquist LM, Weiss KE, Law EF, Sil S, Herbert LJ, Horn SB 2010). In either case, no emphasis was put on well adapted VR game design. We believed that the extended length of the burn dressing change procedure and the repetitive exposure to painful stimuli provide a particular context of care where VR and game design could be highly useful.

Beyond the immediate pain experience, children may develop memories that are accurate, positively or negatively estimated (i.e., remembering more or less pain, respectively, as compared to initial pain reports). The way that children's memories are framed is important for future pain coping and distress at subsequent painful experiences (Noel M, McMurtry CM, Chambers CT, McGrath PJ 2010 - Noel M, Chambers CT, Petter M, McGrath PJ, Klein RM, Stewart SH 2012). Moreover, it has been shown that pharmacological and nonpharmacological pain management strategies may buffer children from developing such 
biases in pain memories (Cohen LL, Blount RL, Cohen RJ, Ball CM, McClellan CB, Bernard RS 2001). Therefore, examining the impact of VR on children's pain memories and expectancies in addition to their experience of pain and fear is important. Those facts brought us to identify two Research Questions and three hypotheses:

Primary Research Question: In children aged 7 to 18 years, who have suffered burn injuries, does VR distraction combined with analgesics provide better pain relief during dressing changes than standard pharmacological treatment alone?

Secondary Research Questions: a) In children aged 7 to 18 years, who have suffered burn injuries, does VR distraction combined with analgesics provide better anxiety relief during dressing change than standard pharmacological treatment alone? b) Does the use of VR distraction lead to less distressing memories of pain than standard pharmacological treatment alone? c) Does the use of VR distraction reduce the dose and side effects of analgesics used during burn wound dressing changes in children?

Hypotheses a) VR distraction combined with analgesics is more effective than standard treatment (analgesics alone) to manage procedural pain (during dressing change) of children with burn injuries.

Hypotheses b) VR distraction combined with analgesics is more effective than standard treatment (analgesics alone) on procedural anxiety (during dressing change) of children with burn injuries.

Hypotheses c) VR distraction combined with analgesics will lead to less distressing memories of pain than standard treatment (analgesics alone) in children with burn injuries.

\section{METHODS}

\subsection{Design.}

One-group within subject design. Given the difficulty of ensuring a single or a doubleblind condition, and in order to limit the interpersonal variability between participants, each child is serve as their own control and are receiving both standard and experimental treatments through a fixed sequential order.

\subsection{Sample and Se tting.}

Participants are beeing recruited through convenience sampling upon admission to the surgical-trauma burn unit at $\mathrm{CHU}$ SteJustine, Montreal (Qc), Canada. Children and their parents are invited to participate in the study if they meet the following criteria: 1) between the ages of 7 to 18 years and suffer from a burn injury requiring wound dressing change, 2 ) presence of a consenting parent who can understand, read and write either French or English. Children are excluded from the study if they meet the following criteria: 1) require intensive care, 2) have a diagnosed cognitive impairment, 3) are unconscious or intubated during dressing change, 4) suffer from epilepsy considering the nature of the intervention, 5) allergic to opioids or other analgesics used for standard pharmacological treatment. For a statistical power of $80 \%$, an alpha of 0.05 , and a significant difference of two points on a 0 to 10 FLACC pain scale, the size of the total desired sample is 40 participants who will serve as their own controls. The setting of the study is the main referral center for pediatric burns in Quebec. It receives nearly 60 admissions during the time of the year when there is a higher incidence of burns (April to October) and over all close to 100 admissions per year. One third of the total admissions are aged 7 years and older. Given a possible census of 33 admissions per year, it appears feasible to recruit around 40 patients over a period of two years. This is also the reason behind the choice of the design which would be the most feasible given the total population available for recruitment.

\subsection{Interventions.}

A) Standard pharmacological treatment consists of opioids and anxiolytics according to the unit's protocol and adjusted to each participant's age, weight and condition by the anesthesiologist and pain clinic nurse. Use of any co-interventions for pain or 
anxiety management (music, comforting, child life specialist or other) is being documented.

B) Virtual reality game distraction through the use of $\mathrm{OR}$ is used as the experimental intervention. The OR is made of two Oled panels with a resolution of $1200 \mathrm{p}$ running at $90 \mathrm{~Hz}$. It has very effective 360 degree positional tracking and integrated 3D audio. These combine to produce a high level of immersion, with high photorealism while maintaining the low latency necessary to induce presence and prevent cybersickness. The latest version also puts an emphasis on ergonomics and user's comfort. The child or a proxy, depending on age and burn site, has the opportunity to interact with the game, although the interactive component is not mandatory for the immersive and distractive experiences. The video game is adapted to each age-group of children recruited and is different from the commercially available ones as each gameplay/level of difficulty is tailored to children's developmental stages and also to their injury. It also allows us to control the speed of movement to avoid cyber sickness. The music provides audio stimulation while allowing the child to relax during the procedure. The game have been tested and approved by a team of healthcare professionals with extensive experience in pediatrics.

\subsection{Measures.}

Pain, anxiety and comfort measures are taken at each study period: $\mathrm{T} 1$, before the procedure (for expectancy and baseline state), T2, immediately after the procedure and T3, 30 minutes after the procedure. Memories of pain/anxiety is assessed at least 24 hours after each procedure. The primary outcome measure is the global pain score measured immediately after the procedure (T2) using the Visual Analogue Scale (VAS) (Scott PJ, Ansell BM, Huskisson EC 1977). The VAS is a widely established measure of pain intensity recommended by the PedIMMPACT group for self-report measures of acute pain intensity in clinical trials in children and adolescents (McGrath
PJ, Walco GA, Turk DC, Dworkin RH, Brown MT, Davidson K 2008). It requires moving a cursor on a standard $100-\mathrm{mm}$ line labeled "no pain" (0) at the left anchor and "worst pain ever" (100) at the right anchor. Secondary outcome measures includes: (a) an observational/ behavioral measure of pain using the Face, Legs, Activity, Cry and Consolability (FLACC) scale assessed by an independent observer (research assistant), (b) a self-report measure of expected and experienced anxiety using the Children`s Fear Scale (CFS) (McMurtry CM, Noel M, Chambers CT, McGrath PJ 2011), (c) a measure of nurses' satisfaction via a questionnaire developed for the pilot study, (d) an average of standard and additional doses of analgesics administered before (T1) and after the procedure (T2-T3), (d) proportion of participants with side effects at $\mathrm{T}-2$ and $\mathrm{T}-3$, (e) time required to perform dressing change in both groups, (f) a measure of comfort during the procedure using the behavioural observation scale of comfort level for child burn victims (OCCEB-BECCO)(Tremblay V, Khadra, C, Charette, S, Boisvert, G, Te, C, Farmer, L, Paquette, J, Leblanc, G, Villeneuve, E, Le May, S 2015), and (g) a measure of the child's memories of pain and anxiety 24 hours after each dressing change procedure using the same measures administered during the dressing change to assess these constructs.

The FLACC is a behavioral/ observational pain scale for children from 0 to 18 years old (von Baeyer CL, Spagrud LJ 2007 - Crellin D, Sullivan TP, Babl FE, O'Sullivan R, Hutchinson A 2007), that includes five separate items as mentioned in its name : Faces, Legs, Activity, Cry and Consolability (Merkel SI, Voepel-Lewis T, Shayevitz JR, Malviya S 1997). Each of the items is scored on a range of 0 to 2 then added to provide a total scoring of pain from 0 to 10 . This scale has been used in studies to assess procedural pain in children (Gee Kee EL, Kimble RM, Cuttle L, Khan A, Stockton KA 2015 - Kim $\mathrm{CH}$, Yoon JU, Lee HJ, Shin SW, Yoon JY, Byeon GJ 2012). 
The CFS is a self-report numerical measure of fear and anxiety in children undergoing painful medical procedures adapted from the Faces Anxiety Scale (McKinley S, Coote K, Stein-Parbury J 2003). This one-item scale shows five sex-neutral faces of varying degrees of fear ranging from 0 (no fear) on the far left to 4 (extremely fearful) on the far right. The measure shows good evidence of interrater and test-retest reliability as well as concurrent and discriminant validity (McMurtry CM, Noel M, Chambers CT, McGrath PJ 2011). The faces are based on the facial muscle changes involved in a fearful expression (Ekman P, Friesen WV 1976).

OCCEB-BECCO is a scale of 0-10, developed by the pain clinic team of the hospital where the study will take place and validated by Tremblay (Tremblay $\mathrm{V}$, Khadra, C, Charette, S, Boisvert, G, Te, C, Farmer, L, Paquette, J, Leblanc, G, Villeneuve, E, Le May, S 2015). It was developed specifically for children with burn injuries. It consists in a rating from 0 to 2 or 3 for different observational items: face, crying, movements and the child's attitude. The validation study of the OCCEB-BECCO scale showed an agreement rate of $90-95 \%$ between experts on all the items in regard to clarity and relevance and an internal consistency of 0.82 (Tremblay V, Khadra, C, Charette, S, Boisvert, G, Te, C, Farmer, L, Paquette, J, Leblanc, G, Villeneuve, E, Le May, S 2015).

Pain Memories: Pain Memories: At least 24 hours after each dressing change, children report on their memories of pain and anxiety using the same measures administered during the dressing change to assess these constructs but framed in terms of recall. Parents of younger children and adolescents are given copies of the rating scales (VAS, CFS) in sealed envelopes at the time of the dressing change procedure and asked to refrain from opening the envelop until the follow-up with the research assistant. During the dressing change session, children and parents are informed that they will be contacted by a research assistant via telephone or in-person (if hospitalised or have a scheduled follow-up visit at the day hospital) but they are unaware that it's for memory assessment/recall purposes in order to avoid potential biases. At the time of the measure of pain/anxiety memories, children or parents are asked to open the envelopes and use the rating scales to report how much pain and anxiety they remember that they felt during the dressing change procedure. Parents would be asked to avoid influencing their child's response to avoid biasing their recall.

\subsection{Study procee dings.}

The study has two-time periods. In the first period, recruited participants receive the standard pharmacological treatment as per unit protocol. In a second period, during the second dressing change procedure, the same participants receive standard pharmacological therapy in combination with VR distraction intervention. This sequence is respected for all participants in order to avoid a possible carryover effect if the VR intervention is administered first. The time between the two study periods vary between 2 to 3 days depending on the need of dressing change, the latter being performed 2 to 3 times per week.

\subsection{Data analysis plan.}

Multivariate Analyses and quantitative comparisons is being conducted within and between subjects for the dependent variables for repeated measures. For each study period, the mean difference in pain scores of patients at each time-period is compared using an ANOVA model for repeated and independent measures. Since the use of rescue medication is a potential indication of a treatment failure, the primary analysis is supplemented by an analysis comparing the proportion of patients receiving rescue medication anytime during the procedure. Interpretation of the primary analyses is made with reference to the data regarding rescue medication use. Data collected on dichotomous variables is analyzed using a 
chi-square test and post-hoc analyses if the results are statistically significant.

\section{KNOWLEDGE TRANSLATION PLAN}

The study will have immediate translatable clinical implications for procedural pain management in children. The members of the surgical-trauma unit have been involved in the project since its early stages. The users' and inpatient's committee was informed about the study. Parents and hospital managers attending this committee have provided the funding to develop the new game for younger children. If the study demonstrates the beneficial effect of VR on pain management, the prototype will remain in the hospital and can be immediately available for use during dressing changes. Healthcare professionals participating in this project will disseminate the results to colleagues allowing a multi-modal knowledge translation plan that will ensure rapid application for the knowledge gained through this study. A manuscript will be submitted to a high impact peer-reviewed journal (e.g. Pediatrics) and results will be presented at pediatric (Canadian Pediatric Society), burn (Canadian Association of Burn Nurses) and pain (International Association for the Study of Pain) meetings. We have already started presenting the protocol to local clinicians and stakeholders during the innovation week at CHU SteJustine. We will also make the research results available through our hospital's online and social media pipelines, forum postings, committee of patients and through the Research Center's 'Café scientifique', evenings attended by parents and professionals. Recruitment of the necessary sample size of patients is somewhat difficult to estimate as cases of burn injuries, similar to traumas, happen in a random fashion. We will have full coverage by research nurses and graduate students during the time of the week for dressing changes, thereby enabling us to recruit the maximum possible group of burn patients referred to the unit. Further, we know that use of virtual reality might cause symptoms similar to those of motion sickness such as nausea, vertigo or vomiting. Our team of experts in immersive technologies have worked on producing games for OR especially to minimize cybersickness.

\section{CONCLUSION}

We hope that the DREAM project will provide evidence-based knowledge on nonpharmacological methods of procedural pain management. The experiment and data analysis is currently taking place and it's already telling us that VR distraction could be a very interesting method to relieve pain, that it's easy to use and has showed very little side effects. We strongly believe it could reduce the need for opioids and anxiolytics in burn children and provide a more humanistic care environment. Expected results should have a direct effect on physical (pain, comfort) and psychological (anxiety) health of the child. In addition, clinical implications may include other indicators of quality of care and economic benefits such as duration of the dressing change, amount of opioids and anxiety drugs administered, use of coanalgesia and duration of wound healing.

\section{REFERENCES}

[1] Ratcliff SL, Brown A, Rosenberg L, Rosenberg M, Robert RS, Cuervo LJ, et al. The effectiveness of a pain and anxiety protocol to treat the acute pediatric burn patient. Burns. 2006;32(5):554-62.

[2] Sharma RK, Parashar A. Special considerations in paediatric burn patients. Indian Journal of Plastic Surgery. 2010;43(Suppl):S43-S50.

[3] von Baeyer CL, Tupper SM. Procedural Pain Management for Children Receiving Physiotherapy. Physiotherapy Canada. 2010;62(4):327-37.

[4] Alencar de Castro RJ, Leal PC, Sakata RK. Pain management in burn patients. Brazilian journal of anesthesiology. 2013;63(1):149-53. 
[5] Gandhi M, Thomson C, Lord D, Enoch S. Management of Pain in Children with Burns. International Journal of Pediatrics. 2010;2010.

[6] Hoffman HG, Meyer WJ, 3rd, Ramirez M, Roberts L, Seibel EJ, Atzori B, et al. Feasibility of articulated arm mounted Oculus Rift Virtual Reality goggles for adjunctive pain control during occupational therapy in pediatric burn patients. Cyberpsychology, behavior and social networking. 2014;17(6):397-401.

[7] Uman LS, Birnie KA, Noel M, Parker JA, Chambers CT, McGrath PJ, et al. Psychological interventions for needlerelated procedural pain and distress in children and adolescents. The Cochrane database of systematic reviews. 2013;10:Cd005179.

[8] Melzack R, Wall PD. Pain mechanisms: a new theory. Science. 1965;150(3699):971-9.

[9] McCaul KD, Malott JM. Distraction and coping with pain. Psychological bulletin. 1984;95(3):516-33.

[10] Li A, Montaño Z, Chen VJ, Gold JI. Virtual reality and pain management: current trends and future directions. Pain management. 2011;1(2):147-57.

[11] Harrison D, Joly C, Chretien C, Cochrane S, Ellis J, Lamontagne C, et al. Pain prevalence in a pediatric hospital: raising awareness during Pain Awareness Week. Pain research \& management. 2014;19(1):e24-30.

[12] Malloy KM, Milling LS. The effectiveness of virtual reality distraction for pain reduction: a systematic review. Clinical psychology review. 2010;30(8):1011-8.

[13] Hoffman HG, Chambers GT, Meyer WJ, 3rd, Arceneaux LL, Russell WJ, Seibel EJ, et al. Virtual reality as an adjunctive non-pharmacologic analgesic for acute burn pain during medical procedures. Annals of behavioral medicine. 2011;41(2):183-91.

[14] Hoffman HG, Richards TL, Van Oostrom T, Coda BA, Jensen MP, Blough DK, et al. The analgesic effects of opioids and immersive virtual reality distraction: evidence from subjective and functional brain imaging assessments. Anesthesia and analgesia. 2007;105(6):1776-83.

[15] Sharar SR, Miller W, Teeley A, Soltani M, Hoffman HG, Jensen MP, et al. Applications of virtual reality for pain management in burn-injured patients. Expert Rev Neurother. 2008;8(11):166774.

[16] Das DA, Grimmer KA, Sparnon AL, McRae SE, Thomas BH. The efficacy of playing a virtual reality game in modulating pain for children with acute burn injuries: a randomized controlled trial [ISRCTN87413556]. BMC pediatrics. 2005;5(1):1.

[17] Asl Aminabadi N, Erfanparast L, Sohrabi A, Ghertasi Oskouei S, Naghili A. The Impact of Virtual Reality Distraction on Pain and Anxiety during Dental Treatment in 4-6 Year-Old Children: a Randomized Controlled Clinical Trial. Journal of dental research, dental clinics, dental prospects. 2012;6(4):117-24.

[18] Birnie KA, Noel M, Parker JA, Chambers CT, Uman LS, Kisely SR, et al. Systematic review and meta-analysis of distraction and hypnosis for needlerelated pain and distress in children and adolescents. Journal of pediatric psychology. 2014;39(8):783-808.

[19] Morris LD, Louw QA, Grimmer-Somers $K$. The effectiveness of virtual reality on reducing pain and anxiety in burn injury patients: a systematic review. The Clinical journal of pain. 2009;25(9):81526.

[20] Dahlquist LM, Weiss KE, Law EF, Sil S, Herbert LJ, Horn SB, et al. Effects of videogame distraction and a virtual reality type head-mounted display helmet on cold pressor pain in young elementary school-aged children. Journal of pediatric psychology. 2010;35(6):617-25.

[21] Noel M, McMurtry CM, Chambers CT, McGrath PJ. Children's memory for painful procedures: the relationship of pain intensity, anxiety, and adult 
behaviors to subsequent recall. Journal of pediatric psychology. 2010;35(6):626-36.

[22] Noel M, Chambers CT, Petter M, McGrath PJ, Klein RM, Stewart SH. Pain is not over when the needle ends: A review and preliminary model of acute pain memory development in childhood. Pain management. 2012;2(5):487-97.

[23] Cohen LL, Blount RL, Cohen RJ, Ball $\mathrm{CM}$, McClellan CB, Bernard RS. Children's expectations and memories of acute distress: short- and long-term efficacy of pain management interventions. Journal of pediatric psychology. 2001;26(6):367-74.

[24] Scott PJ, Ansell BM, Huskisson EC. Measurement of pain in juvenile chronic polyarthritis. Ann Rheum Dis. 1977;36(2):186-7.

[25] McGrath PJ, Walco GA, Turk DC, Dworkin RH, Brown MT, Davidson K, et al. Core outcome domains and measures for pediatric acute and chronic/recurrent pain clinical trials: PedIMMPACT recommendations. The journal of pain. 2008;9(9):771-83.

[26] McMurtry CM, Noel M, Chambers CT, McGrath PJ. Children's fear during procedural pain: preliminary investigation of the Children's Fear Scale. Health psychology. 2011;30(6):780-8.

[27] Tremblay V, Khadra, C, Charette, S, Boisvert, G, Te, C, Farmer, L, Paquette, J, Leblanc, G, Villeneuve, E, Le May, S. Development of a scale to measure comfort in children with burn injuries (poster). 10th International Forum on Pediatric Pain; 2015; White Point Beach Resort, Nova Scotia, Canada.

[28] von Baeyer CL, Spagrud LJ. Systematic review of observational (behavioral) measures of pain for children and adolescents aged 3 to 18 years. Pain. 2007;127(1-2):140-50.

[29] Crellin D, Sullivan TP, Babl FE, O'Sullivan R, Hutchinson A. Analysis of the validation of existing behavioral pain and distress scales for use in the procedural setting. Paediatr Anaesth. 2007;17(8):720-33.

[30] Merkel SI, Voepel-Lewis T, Shayevitz JR, Malviya S. The FLACC: a behavioral scale for scoring postoperative pain in young children. Pediatric nursing. 1997;23(3):293-7.

[31] Gee Kee EL, Kimble RM, Cuttle L, Khan A, Stockton KA. Randomized controlled trial of three burns dressings for partial thickness burns in children. Burns. 2015;41(5):946-55.

[32] Kim CH, Yoon JU, Lee HJ, Shin SW, Yoon JY, Byeon GJ. Availability of a $5 \%$ lidocaine patch used prophylactically for venipuncture- or injection-related pain in children. Journal of anesthesia. 2012;26(4):552-5.

[33] McKinley S, Coote K, Stein-Parbury J. Development and testing of a Faces Scale for the assessment of anxiety in critically ill patients. Journal of advanced nursing. 2003;41(1):73-9.

[34] Ekman P, Friesen WV. Measuring facial movement. Environmental psychology and nonverbal behavior. 1976;1(1):5675.

[35] Ekman P, Friesen WV. Measuring facial movement. Environmental psychology and nonverbal behavior. 1976;1(1):5675. 